Original research article

\title{
Comparison of dystonia between Parkinson's disease and atypical parkinsonism: The clinical usefulness of dystonia distribution and characteristics in the differential diagnosis of parkinsonism
}

\author{
Won Tae Yoon * \\ Department of Neurology, Kangbuk Samsung Hospital, Sungkyunkwan University School of Medicine, Republic of Korea
}

\section{A R T I C L E I N F O}

Article history:

Received 4 May 2017

Accepted 5 November 2017

Available online 14 November 2017

\section{Keywords:}

Dystonia

Parkinson's disease

Atypical parkinsonism

Multiple system atrophy

Progressive supranuclear palsy

Corticobasal degeneration

\begin{abstract}
A B S T R A C T
Objective: Dystonia is occasionally found in patients with Parkinson's disease (PD) and atypical parkinsonisms. However, systematic comparative analysis of the association between dystonia and parkinsonism have seldom been reported. The goals of this study are to compare the clinical characteristics and distributions of dystonia between PD, multiple system atrophy (MSA), progressive supranuclear palsy (PSP) and corticobasal degeneration (CBD).

Methods: We prospectively enrolled 176 patients who presented with dystonia and parkinsonism out of 1278 patients with parkinsonism. We analyzed the clinical features of dystonia and parkinsonism.

Results: The frequencies of dystonia were $11.0 \%$ in PD, 20.9\% in MSA, 40.7\% in PSP and $66.7 \%$ in CBD. Dystonia symptoms were most frequent in CBD and relatively more frequent in PSP and MSA $(p<0.001)$. Moreover, multiple types of dystonia occurred most frequently in MSA $(p=0.034)$. According to the distribution of dystonia, cranio-facial dystonia (CFD) and cervical dystonia (CD) were more frequently observed in atypical parkinsonism $(p=0.001)$. In contrast, limb dystonia (LD) was more frequently observed in both PD and CBD, and truncal dystonia (TD) was more frequently detected in $\mathrm{PD}(p<0.001)$. Levodopa medication related dystonia was markedly more frequent in PD than in atypical parkinsonism $(p=0.030)$.

Conclusions: In this long-term, observational, prospective study, we concluded that levodopa medication related LD and TD were more frequently observed in PD than in atypical parkinsonism. Conversely, levodopa medication non-related CFD and CD were more frequently observed in atypical parkinsonism, and coexisting of some types of multiple dystonia may be unique features of atypical parkinsonism. TD or multiple types of LD, might be representative of $\mathrm{PD}$ rather than atypical parkinsonism.
\end{abstract}

(C) 2017 Published by Elsevier Sp. z o.o. on behalf of Polish Neurological Society.

\footnotetext{
* Correspondence to: Department of Neurology, Kangbuk Samsung Hospital, Sungkyunkwan University School of Medicine, \#29 Saemunan-ro, Jongno-gu, Seoul 03181, Republic of Korea.

E-mail addresses: wtyoon@skku.edu, wtyoon@gmail.com

https://doi.org/10.1016/j.pjnns.2017.11.004

0028-3843/@ 2017 Published by Elsevier Sp. z o.o. on behalf of Polish Neurological Society.
} 


\section{Introduction}

Dystonia is a syndrome that is characterized by sustained muscle contraction associated with twisting, repetitive, and patterned movements, abnormal posture, or both [1]. Dystonia may coexist with parkinsonism in a number of neurodegenerative, genetic, toxic, and metabolic disorders of which Parkinson's disease (PD) and atypical parkinsonisms are the most common [2,3]. Clinical associations between and dystonia and various parkinsonisms have been reported in some studies [4-7]. Although rare, one such study also reported the overall frequency and clinical features of dystonia in atypical parkinsonism [8].

However, the clinical relationships remain unclear; for example, one study commented that the true frequency and range of dystonia manifestations remain unknown in autopsy-proven cases of parkinsonism, including multiple system atrophy (MSA), progressive supranuclear palsy (PSP), corticobasal degeneration (CBD) and $\mathrm{PD}$ [9], and systematic prospective, comparative analysis of the clinical features of dystonia associated with parkinsonism have seldom been reported.

The aims of the present study were to investigate the clinical characteristics of dystonia associated with parkinsonism in a larger patient sample than that in previous reports and compare these characteristics among the various types of parkinsonism to identify the clinical usefulness of the distributive pattern of dystonia in the differential diagnosis of parkinsonism.

\section{Materials and methods}

Between January 2002 to December 2004, we investigated 185 consecutive patients who presented with dystonia combined with parkinsonism. Among these patients, we prospectively enrolled 176 patients who presented with dystonia combined with parkinsonism out of 1278 patients with parkinsonism registered at the Movement Disorder Clinic of the Samsung Medical Center during the same period, from January 2002 to December 2004. We included patients with long-term followup data over five years (to December 2009), full evaluations for parkinsonism and clinical diagnoses confirmed by at least two specialist neurologists from movement disorder clinics. Under the established diagnostic criteria [10-15], 1278 patients with parkinsonism were divided into 1045 patients with $\mathrm{PD}, 191$ patients with MSA, 27 patients with PSP and 15 patients with CBD. Moreover, we excluded 9 patients with uncertain diagnosis of parkinsonism $(n=2)$, those lost to follow-up $(n=2)$ and those with combined dystonia due to medical or neurological reasons other than parkinsonism with the following other diagnosis: dementia with Lewy bodies (DLB) $(n=2)$, normal pressure hydrocephalus (NPH) $(n=1)$, metabolic encephalopathy $(n=1)$, and post-stroke parkinsonism with dystonia $(n=1)$

We clinically diagnosed dystonia using the established definition $[2,16]$ and analyzed the clinical features of dystonia and parkinsonism, including demographics, the temporal profiles of the symptoms, the distribution and characteristics of dystonia, the clinical diagnosis of parkinsonism and the relationship with levodopa treatment. Dystonia was subclassified as cranio-facial dystonia (CFD), cervical dystonia (CD), truncal dystonia (TD) and limb dystonia (LD) according to the distribution of dystonia among the enrolled parkinsonism patients, and we also clinically examined cases of unusual dystonia phenotypes, such as dystonia preceding parkinsonism (Pre-Dystonia) and clinically dominant dystonia if there were multiple coincidently emerging variant types of dystonia (Multi-Dystonia), as occurred in one parkinsonism patient.

All data are presented as the mean \pm standard error of the mean (SEM). The PASW (Version 22.0; IBM SPSS Inc., Chicago, IL) program for Windows was used for statistical analysis. The baseline demographic and clinical data were analyzed by Student's t-test, and data regarding sex differences and the number of subjects with the presence of dystonia between each group were analyzed using the chi-square test. Data related to age of onset, the interval from onset to parkinsonism, and the number of characteristics regarding dystonic symptoms were analyzed using one-way analysis of variance (one-way ANOVA). Fisher's exact test was used to evaluate the difference in the number of dystonias between each group, and the statistical significance level was set at $p<0.05$.

\section{Results}

Among 176 dystonic patients with parkinsonism, 115 patients were clinically diagnosed with $\mathrm{PD}, 40$ were probable MSA, 11 were probable PSP, and 10 were probable CBD. Fig. 1 shows the patient flow, including information about 9 excluded patients. Demographic and clinical profiles of the dystonic patients in each parkinsonism group are presented in Table 1 . Among the total 1278 parkinsonism patients (1045 PD, $191 \mathrm{MSA}, 27 \mathrm{PSP}$ and 15 CBD), the frequencies of dystonia among each parkinsonism type were $11.0 \%(115 / 1045)$ in $\mathrm{PD}, 20.9 \%$ (40/191) in MSA, $40.7 \%$ (11/27) in PSP and 66.7\% (10/15) in CBD. Overall, dystonia symptoms were more frequent in CBD and PSP than in PD $(p<0.001)$, and in terms of sex differences, dystonia was more frequent in men than in women among PSP patients $(p=0.019)$. The onset age of initial dystonic symptoms was older in CBD and younger in PD $(p<0.001)$.

In CBD patients, only limb dystonic symptoms without other types of dystonia were observed, with no unusual PreDystonia phenotypes. Despite the small number of patients, Multi-Dystonia was relatively more frequent in MSA than in PD or PSP $(p=0.034)$. Aside from these findings, no other significant clinical demographic differences were observed among the various types of parkinsonism (Table 1 ).

According to the distribution of dystonia, CFD and CD were more frequently observed in atypical parkinsonism (39/61, $63.9 \%$ and $7 / 61,11.5 \%$, respectively) than in $\mathrm{PD}(16 / 115,13.9 \%$ and $1 / 115,0.9 \%$, respectively) $(p<0.001)$. In contrast, TD and LD were more frequently observed in $\operatorname{PD}(32 / 115,27.8 \%$ and $66 /$ $115,57.4 \%$, respectively) than in atypical parkinsonism $(2 / 61$, $3.3 \%$ and $13 / 61,21.3 \%$, respectively) ( $p=0.001)$. TD, including camptocormia, was detected only in PD and levodopa medication non-related MSA $(p<0.001)$. In terms of the relationship with levodopa treatment, levodopa medication related dystonia was markedly more frequent in PD than in 


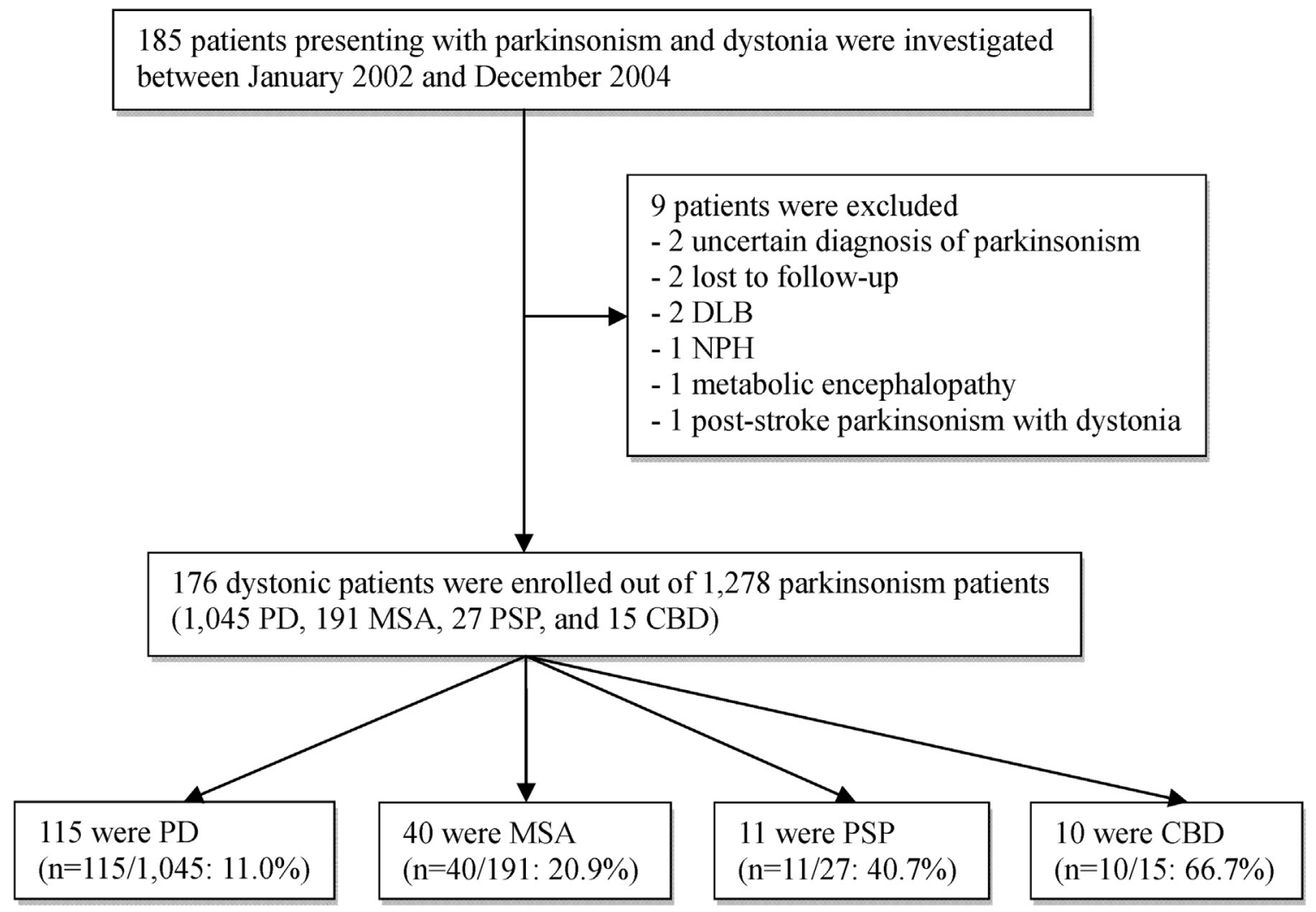

Fig. 1 - Patient flow. DLB, dementia with Lewy bodies; NPH, normal pressure hydrocephalus; PD, Parkinson's disease; MSA, multiple system atrophy; PSP, progressive supranuclear palsy; CBD, corticobasal degeneration.

\section{Table 1 - Demographic and clinical profiles of dystonic patients in each type of parkinsonism.}

\begin{tabular}{|c|c|c|c|c|c|}
\hline Patients & $\mathrm{PD}^{\mathrm{a}}$ & $\mathrm{MSA}^{\mathrm{b}}$ & $\mathrm{PSP}^{\mathrm{C}}$ & $\mathrm{CBD}^{\mathrm{d}}$ & $p$ Value \\
\hline Dystonia/total patients $(\mathrm{N})$ & $115 / 1045$ & $40 / 191$ & $11 / 27$ & $10 / 15$ & $<0.001$ \\
\hline (Prevalence of dystonia, \%) & $(11.0 \%)$ & $(20.9 \%)$ & $(40.7 \%)$ & $(66.7 \%)$ & \\
\hline $\operatorname{Sex}(M / F)$ & $41 / 74$ & $19 / 21$ & $9 / 2$ & $5 / 5$ & 0.019 \\
\hline Onset age (y): initial symptom & $54.6 \pm 10.8$ & $60.8 \pm 9.0$ & $59.8 \pm 4.1$ & $67.1 \pm 7.7$ & $<0.001$ \\
\hline Onset interval $(\mathrm{m})$ : to dystonia & $54.9 \pm 45.6$ & $41.9 \pm 35.6$ & $47.5 \pm 43.5$ & $69.0 \pm 28.7$ & 0.217 \\
\hline Multiple types of dystonia (N) & 12 & 18 & 5 & 0 & 0.034 \\
\hline Preceding dystonia to parkinsonism $(\mathrm{N})$ & 8 & 5 & 2 & 0 & $\mathrm{NA}^{\mathrm{e}}$ \\
\hline $\begin{array}{l}\text { a Parkinson's disease (PD). } \\
\text { b Multiple system atrophy (MSA). } \\
\text { c Progressive supranuclear palsy (PSP). } \\
\text { d Corticobasal degeneration (CBD). } \\
\text { e Not available (NA). }\end{array}$ & & & & & \\
\hline
\end{tabular}

atypical parkinsonism $(p=0.034)$. Although TD was more frequent in $\mathrm{PD}$ regardless of the relationship with levodopa medication, CFD and CD were more frequent in levodopa medication non-related atypical parkinsonism $(p<0.001)$, and LD was more frequent in levodopa medication related $\mathrm{PD}$, as shown in Table $2(p=0.030)$.

Among the 35 patients with multiple types of dystonia, twenty-three were diagnosed with atypical parkinsonism, and twelve were diagnosed with PD. According to the subclasses of the combinations of multiple types of dystonia, combination groups with two-variable CFD or CFD with CD were observed only in atypical parkinsonism, and other combination groups of CD with LD, TD with LD and two-variable LD were observed only in PD (Table 3). Excluding some small groups of patients, the two major multiple-type dystonias were two-variable CFD $(19 / 35,54.3 \%)$ and two-variable $\operatorname{LD}(7 / 35,20 \%)$, with all patients in the former group being diagnosed as atypical parkinsonism (15 MSA and 4 PSP) and all patients in the latter group being diagnosed as PD with significant clinical and demographic differences $(p<0.001)$. 
Table 2 - Distribution of dominant dystonia in each parkinsonism patient according to the relationship with levodopa medication.

\begin{tabular}{|c|c|c|c|c|c|c|}
\hline Parkinsonism & $\mathrm{PD}^{\mathrm{a}}$ & $\begin{array}{c}\text { Atypical } \\
\text { parkinsonism (AP) }\end{array}$ & $\mathrm{MSA}^{\mathrm{b}}$ & $\mathrm{PSP}^{\mathrm{C}}$ & $\mathrm{CBD}^{\mathrm{d}}$ & $\begin{array}{c}p \text {-Value } \\
\text { PD vs. AP/All groups }\end{array}$ \\
\hline Total number of dystonic patients $(\mathrm{N})$ & 115 & 61 & 40 & 11 & 10 & $<0.001 / 0.001$ \\
\hline $\mathrm{CFD}^{\mathrm{e}}(\mathrm{N})$ & 16 & 39 & 31 & 8 & 0 & \\
\hline $\mathrm{CD}^{\mathrm{f}}(\mathrm{N})$ & 1 & 7 & 5 & 2 & 0 & \\
\hline $\mathrm{TD}^{\mathrm{g}}(\mathrm{N})$ & 32 & 2 & 2 & 0 & 0 & \\
\hline $\mathrm{LD}^{\mathrm{h}}(\mathrm{N})$ & 66 & 13 & 2 & 1 & 10 & \\
\hline Levodopa medication non-related & 58 & 55 & 35 & 11 & 9 & $<0.001 /<0.001$ \\
\hline $\mathrm{CFD}^{\mathrm{e}}(\mathrm{N})$ & 6 & 36 & 28 & 8 & 0 & \\
\hline$C D^{f}(N)$ & 0 & 6 & 4 & 2 & 0 & \\
\hline $\mathrm{TD}^{\mathrm{g}}(\mathrm{N})$ & 24 & 2 & 2 & 0 & 0 & \\
\hline $\mathrm{LD}^{\mathrm{h}}(\mathrm{N})$ & 28 & 11 & 1 & 1 & 9 & \\
\hline Levodopa medication related & 57 & 6 & 5 & 0 & 1 & $0.034 / 0.030$ \\
\hline $\mathrm{CFD}^{\mathrm{e}}(\mathrm{N})$ & 10 & 3 & 3 & 0 & 0 & \\
\hline$C D^{f}(N) f$ & 1 & 1 & 1 & 0 & 0 & \\
\hline $\mathrm{TD}^{\mathrm{g}}(\mathrm{N})$ & 8 & 0 & 0 & 0 & 0 & \\
\hline $\mathrm{LD}^{\mathrm{h}}(\mathrm{N})$ & 38 & 2 & 1 & 0 & 1 & \\
\hline $\begin{array}{l}\text { a Parkinson's disease (PD). } \\
\text { b Multiple system atrophy (MSA). } \\
\text { c Progressive supranuclear palsy (PSP). } \\
\text { d Corticobasal degeneration (CBD). } \\
\text { e Cranio-facial dystonia (CFD). } \\
\text { f Cervical dystonia (CD). } \\
\text { g Truncal dystonia (TD). } \\
\text { h Limb dystonia (CBD). }\end{array}$ & & & & & & \\
\hline
\end{tabular}

\section{Table 3 - Distribution of multiple types of dystonia in each type of parkinsonism.}

\begin{tabular}{|c|c|c|c|c|c|c|}
\hline \multirow[t]{2}{*}{ Parkinsonism } & \multirow[t]{2}{*}{$\mathrm{PD}^{\mathrm{a}}$} & \multirow[t]{2}{*}{$\begin{array}{c}\text { Atypical } \\
\text { parkinsonism (AP) }\end{array}$} & \multirow[t]{2}{*}{$\mathrm{MSA}^{\mathrm{b}}$} & \multirow[t]{2}{*}{$\mathrm{PSP}^{\mathrm{C}}$} & \multirow[t]{2}{*}{$\mathrm{CBD}^{\mathrm{d}}$} & \multirow{2}{*}{$\begin{array}{c}p \text {-Value } \\
\text { PD vs. AP/All groups }\end{array}$} \\
\hline & & & & & & \\
\hline $\begin{array}{l}\text { Total number of patients with } \\
\text { multiple dystonias }(\mathrm{N})\end{array}$ & 12 & 23 & 18 & 5 & 0 & $<0.001 /<0.001$ \\
\hline \multicolumn{7}{|l|}{ Multiple types of dystonia } \\
\hline $\mathrm{CFD}^{\mathrm{e}}+\mathrm{CFD}^{\mathrm{e}}(\mathrm{N})$ & 0 & 19 & 15 & 4 & 0 & \\
\hline$C F D^{e}+C^{f}(N)$ & 0 & 2 & 1 & 1 & 0 & \\
\hline $\mathrm{CFD}^{\mathrm{e}}+\mathrm{TD}^{\mathrm{g}}(\mathrm{N})$ & 1 & 1 & 1 & 0 & 0 & \\
\hline $\mathrm{CFD}^{\mathrm{e}}+\mathrm{LD}^{\mathrm{h}}(\mathrm{N})$ & 1 & 1 & 1 & 0 & 0 & \\
\hline $\mathrm{CD}^{\mathrm{f}}+\mathrm{TD}^{\mathrm{g}}(\mathrm{N})$ & 0 & 0 & 0 & 0 & 0 & \\
\hline$C D^{f}+L D^{h}(N)$ & 1 & 0 & 0 & 0 & 0 & \\
\hline $\mathrm{TD}^{\mathrm{g}}+\mathrm{LD}^{\mathrm{h}}(\mathrm{N})$ & 2 & 0 & 0 & 0 & 0 & \\
\hline$L D^{h}+L D^{h}(N)$ & 7 & 0 & 0 & 0 & 0 & \\
\hline
\end{tabular}

\footnotetext{
a Parkinson's disease (PD).

b Multiple system atrophy (MSA).

c Progressive supranuclear palsy (PSP).

d Corticobasal degeneration (CBD).

e Cranio-facial dystonia (CFD).

f Cervical dystonia (CD).

g Truncal dystonia (TD).

h Limb dystonia (CBD).
}

\section{Discussions}

In this long-term, observational study, we prospectively surveyed dystonia in patients with parkinsonism. In cases with coexisting dystonia and parkinsonism, we retrospectively analyzed the distribution and characteristics of dystonia and prospectively investigated the clinical pattern of dystonia, the relationship between dystonia and levodopa treatment, and the long-term prognosis to confirm the clinical diagnosis.

Parkinsonism and dystonia may coexist not only in neurodegenerative disorders such as PD, MSA, PSP and CBD but also in a number of genetic, toxic and metabolic disorders and as a result of structural lesions in the basal ganglia [2]. Some reports have described the characteristics of the 
dystonias of each single disease entity, groups of atypical parkinsonism or $\mathrm{PD}[4,6-8,17,18]$. In most of these previous studies, the overall frequency of dystonia was found to be relatively high in atypical parkinsonism. However, few studies have compared PD and each atypical parkinsonism. Furthermore, no descriptions regarding the presence of multiple other types of dystonia and preceding dystonia parkinsonism are available, except in some cases reports $[19,20]$.

In terms of the symptoms of each dystonic, previous clinical studies have reported the clinical subtypes of dystonia in parkinsonism. For example, blepharospasm, a type of CFD, is common and more prevalent in atypical parkinsonism than in $\mathrm{PD}[21,22]$. Although these studies are rare, one recent study reported the clinical subtypes of anterocollis in parkinsonian syndromes, finding no other significant clinical differences between the clinical subtypes of anterocollis or primary diagnosis, with the exception that patients with atypical parkinsonian syndromes had more advanced motor symptoms [23]. Another study reported on reversible Pisa syndrome in PD patients on dopaminergic therapy. Similar to the results of our study, a clinically important association between Pisa syndrome as a TD in patients with $\mathrm{PD}$ and dopaminergic therapy was found [24].

In general, PD is associated with the loss of midbrain dopamine neurons [25], and parkinsonism and dystonia have been reported to occur subsequent to strokes in various sites in the basal ganglia, including the striatum and globus pallidus [26-29]. However, some other studies have revealed that dystonia and parkinsonism might be associated with other focal brain lesions or brainstem lesions [30,31]. For these reasons, the co-existence of dystonia and parkinsonism usually results from multiple lesions or the involvement of more than one pathway [2], and one recent animal study reported that parkinsonism and dystonia are differentially induced by the modulation of different territories of the basal ganglia [32]. The pathophysiology and mechanism underlying the co-existence of parkinsonism and dystonia remain unknown, but clinical presentations of dystonia in PD and atypical parkinsonism are relatively common, and dystonia among the clinical set of symptoms and signs associated with parkinsonism may have diagnostic value in the differential diagnosis of parkinsonism $[2,22]$.

In terms of the relationship between dystonia and levodopa treatment, we also found that dystonia may have occurred as an 'off' symptom or as a 'peak-dose' effect of levodopa treatment. Dystonia that is improved by levodopa treatment is more strongly related to $\mathrm{PD}$, while some dystonic symptoms that are aggravated by levodopa treatment are more strongly related to atypical parkinsonism. Moreover, in terms of the relationship between dystonia preceding and following parkinsonism, we also found that some preceding dystonia may occur as a prodromal symptom of parkinsonism, and preceding dystonia is more strongly related to atypical parkinsonism. However, these two suggestions were not statistically confirmed because the number of patients was small. Additionally, we could not assess the history of other medications, mainly including dopamine agonists and anticholinergics, which would have potential effects on dystonia in parkinsonism patients. This point is more likely to be encountered in the clinic, and this issue will be a good subject for clinical studies of differential diagnoses in parkinsonism if prospective observations are performed in the future.

According to our experience during the progression of this study, most cases of CFD and CD were treated with botulinum toxin injection, and most cases of TD and LD were treated with symptomatic medications, including muscle relaxants or anticholinergics and changes in levodopa medication dose. Even though these treatment results regarding dystonic symptoms in all groups were not statistically evaluated because of too many variable factors, many CFD patients treated with botulinum toxin injection might have a good prognosis. Additionally, compared with CD and TD patients, many LD patients treated with medications might have a relatively good prognosis regardless of the methods of treatment.

This study had several limitations. First, even though patient enrollment was conducted in a prospective study design, after enrollment, the clinical data of some patients regarding dystonia and parkinsonism were retrospectively corrected using the patients' direct explanations and subjective history taking. However, the clinical features of the presence of a single type or multiple types of dystonia and the comparative prevalence of dystonia among all study subjects were systemically evaluated. Second, we conducted this study based on only clinical diagnosis of parkinsonism. Clinicopathologic evaluations of the correlation between dystonia and parkinsonism and confirmation of neuropathological diagnosis would be needed to disclose the relationship between dystonic manifestations and pathological substrates of each type of parkinsonism. Third, the pattern and distribution of dystonia and dystonia's co-existence with parkinsonism were assessed for this study. The severity and frequency of dystonia measured by a clinical scale regarding dystonia and the frequency and average of levodopa medication dose were not assessed in detail, which should be considered in future comprehensive studies. Fourth, in addition to levodopa medications, other medications, including dopamine agonists, antipsychotic medications, antidepressants, and symptomatic medications for the treatment of dystonia, were not assessed in detail. However, in this study, relationships between levodopa medication and dystonia were critically observed in all study subjects during the long-term follow-up period regardless of other medications. Fifth, we could not evaluate the previous history of other neurological disorders and medical problems, which could possibly affect the dystonic symptoms or could be hidden causative factors. Future studies with well-designed, detailed evaluations of all factors and long-term comparison between dystonia and parkinsonism are needed to solve these fundamental issues.

\section{Conclusions}

In summary, this study demonstrated that the symptoms of dystonia were most frequent in CBD and more frequent in atypical parkinsonism (e.g., CBD, PSP and MSA, in descending order of frequency) than in PD. Moreover, levodopa medication non-related CFD including blepharospasm or CD, were more frequently observed in atypical parkinsonism (MSA and PSP), 
and some types of multiple combined dystonias (multiple types of CFD or CFD with CD) may be a unique feature of atypical parkinsonism. Conversely, all TD and LD groups were more frequently observed in PD than in atypical parkinsonism, regardless of levodopa medication. TD, including camptocormia or multiple types of LD, might therefore be representative of PD rather than atypical parkinsonism. Moreover, levodopa medication related LD and TD presenting as 'off' symptoms, 'peak-dose' effects or levodopa responsiveness might be representative of $\mathrm{PD}$ and not atypical parkinsonism.

\section{Conflict of interest}

The authors have no financial or personal relations that could pose a conflict of interest.

\section{Acknowledgements}

This manuscript is dedicated to the loving memory of Won Yong Lee (1961-2011), who designed and conducted this study.

\section{Ethics}

The work described in this article was performed in accordance with the Code of Ethics of the World Medical Association (the Declaration of Helsinki) for experiments involving humans and the Uniform Requirements for Manuscripts Submitted to Biomedical journals.

\section{R E F E R E N C E S}

[1] Fahn S, Bressman SB, Marsden CD. Classification of dystonia. Adv Neurol 1998;78:1-10.

[2] Jankovic J, Tintner R. Dystonia and parkinsonism. Parkinsonism Relat Disord 2001;8:109-21.

[3] LeWitt PA, Burns RS, Newman RP. Dystonia in untreated parkinsonism. Clin Neuropharmacol 1986;9:293-7.

[4] Tolosa E, Compta Y. Dystonia in Parkinson's disease. J Neurol 2006;253(Suppl. 7):VII7-13.

[5] Riley DE. Dystonia in multiple system atrophy. J Neurol Neurosurg Psychiatry 2002;72:286.

[6] Vanek Z, Jankovic J. Dystonia in corticobasal degeneration. Mov Disord 2001;16:252-7.

[7] Barclay CL, Lang AE. Dystonia in progressive supranuclear palsy. J Neurol Neurosurg Psychiatry 1997;62:352-6.

[8] Godeiro-Junior C, Felicio AC, Barsottini OG, Aguiar PM, Silva $\mathrm{SM}$, Borges V, et al. Clinical features of dystonia in atypical parkinsonism. Arq Neuropsiquiatr 2008;66:800-4.

[9] Rivest J, Quinn N, Marsden CD. Dystonia in Parkinson's disease, multiple system atrophy, and progressive supranuclear palsy. Neurology 1990;40:1571-8.

[10] Lepore FE, Duvoisin RC. "Apraxia" of eyelid opening: an involuntary levator inhibition. Neurology 1985;35:423-7.

[11] Jankovic J, Havins WE, Wilkins RB. Blinking and blepharospasm. Mechanism, diagnosis, and management. JAMA 1982;248:3160-4.

[12] Obeso JA, Artieda J, Marsden CD. Stretch reflex blepharospasm. Neurology 1985;35:1378-80.
[13] Gelb DJ, Oliver E, Gilman S. Diagnostic criteria for Parkinson disease. Arch Neurol 1999;56:33-9.

[14] Litvan I, Agid Y, Calne D, Campbell G, Dubois B, Duvoisin $\mathrm{RC}$, et al. Clinical research criteria for the diagnosis of progressive supranuclear palsy (Steele-RichardsonOlszewski syndrome): report of the NINDS-SPSP international workshop. Neurology 1996;47:1-9.

[15] Gilman S, Low PA, Quinn N, Albanese A, Ben-Shlomo Y, Fowler CJ, et al. Consensus statement on the diagnosis of multiple system atrophy. J Neurol Sci 1999;163:94-8.

[16] Fahn S. Concept and classification of dystonia. Adv Neurol 1988;50:1-8.

[17] Stamelou M, Alonso-Canovas A, Bhatia KP. Dystonia in corticobasal degeneration: a review of the literature on 404 pathologically proven cases. Mov Disord 2012;27:696-702.

[18] Boesch SM, Wenning GK, Ransmayr G, Poewe W. Dystonia in multiple system atrophy. J Neurol Neurosurg Psychiatry 2002;72:300-3.

[19] Yasuda C, Takei T, Uozumi T, Toyota T, Yuhi T, Adachi H. A case of Parkinson's disease following dystonia. Rinsho Shinkeigaku 2016;56:600-4.

[20] Bruno MK, Ravina B, Garraux G, Hallett M, Ptacek L, Singleton A, et al. Exercise-induced dystonia as a preceding symptom of familial Parkinson's disease. Mov Disord 2004;19:228-30.

[21] Rana AQ Kabir A, Dogu O, Patel A, Khondker S. Prevalence of blepharospasm and apraxia of eyelid opening in patients with parkinsonism, cervical dystonia and essential tremor. Eur Neurol 2012;68:318-21.

[22] Yoon WT, Chung EJ, Lee SH, Kim BJ, Lee WY. Clinical analysis of blepharospasm and apraxia of eyelid opening in patients with parkinsonism. J Clin Neurol 2005;1:159-65.

[23] Revuelta GJ, Benatar M, Freeman A, Wichmann T, Jinnah HA, DeLong MR, et al. Clinical subtypes of anterocollis in parkinsonian syndromes. J Neurol Sci 2012;315:100-3.

[24] Cannas A, Solla P, Floris G, Tacconi P, Serra A, Piga M, et al. Reversible Pisa syndrome in patients with Parkinson's disease on dopaminergic therapy. J Neurol 2009;256:390-5.

[25] Jellinger KA. Post mortem studies in Parkinson's disease-is it possible to detect brain areas for specific symptoms? J Neural Transm Suppl 1999;56:1-29.

[26] Zhang CL, Yin F, He F, Gai N, Shi ZQ, Peng J. A childhoodonset rapid-onset dystonia parkinsonism family with ATP1A3 gene mutation and literatures review. Zhonghua Er Ke Za Zhi 2017;55:288-93.

[27] Kuoppamaki M, Rothwell JC, Brown RG, Quinn N, Bhatia KP, Jahanshahi M. Parkinsonism following bilateral lesions of the globus pallidus: performance on a variety of motor tasks shows similarities with Parkinson's disease. J Neurol Neurosurg Psychiatry 2005;76:482-90.

[28] Munchau A, Mathen D, Cox T, Quinn NP, Marsden CD, Bhatia KP. Unilateral lesions of the globus pallidus: report of four patients presenting with focal or segmental dystonia. J Neurol Neurosurg Psychiatry 2000;69:494-8.

[29] Bhatia KP, Marsden CD. The behavioural and motor consequences of focal lesions of the basal ganglia in man. Brain 1994;117(Pt 4):859-76.

[30] Kostic VS, Stojanovic-Svetel M, Kacar A. Symptomatic dystonias associated with structural brain lesions: report of 16 cases. Can J Neurol Sci 1996;23:53-6.

[31] Pechadre JC, Larochelle L, Poirier LJ. Parkinsonian akinesia, rigidity and tremor in the monkey. Histopathological and neuropharmacological study. J Neurol Sci 1976;28:147-57.

[32] Kumbhare D, Holloway KL, Baron MS. Parkinsonism and dystonia are differentially induced by modulation of different territories in the basal ganglia. Neuroscience 2017;353:42-57. 